\title{
Características laborales del médico joven egresado de la Facultad de Medicina San Fernando *
}

\author{
Moisés Huamán ${ }^{1}$, Kelly Kamimura ${ }^{1}$, Julio Medina ${ }^{2}$, Carlos Bustíos ${ }^{2}$, Elsy Miní ${ }^{2}$, \\ Miguel Benito $^{2}$, César Gutierrez ${ }^{2}$
}

Resumen

Palabras clave

\begin{abstract}
Objetivo: Determinar las características laborales del médico joven egresado de la Facultad de Medicina San Fernando. Diseño: Estudioobservacional, transversal. Lugar: Facultad de Medicina San Fernando, Universidad Nacional Mayor de San Marcos. Participantes: Médicos egresados de la Facultad de Medicina. Intervenciones: Se recolectó datos sociodemográficos, del desempeño laboral, grado profesional, nivel de satisfacción y desempeño extracurricular del pregrado, en 232 médicos egresados de la Facultad de Medicina San Fernando entre 1994 y 2003, elegidos al azar. La recolección se llevó a efecto mediante entrevista directa, telefónica o cuestionario vía correo electrónico. También se obtuvo las notas finales del pregrado de los sujetos. Principales medidas de resultados: Índice de empleo, tipo de labor, desempeño, especialización. Resultados: El 72,4\% estuvo representado por varones, 73,2\% radicaba en Lima, 19,3\% en provincias y 7,5\% en el extranjero; 97\% tenía trabajo remunerado, 51,4\% de ellos tenía 2 o más trabajos; $71 \%$ trabajaba en el sector público. El tipo de labor predominante fue el asistencial; 78,8\% alcanzaba el desempeño laboral esperado; 64,9\% era especialista o estaba en proceso de especialización; 15,4\% tenía o estudiaba una maestría. No se encontró relación significativa entre factores del pregrado y las características laborales o profesionales. Conclusiones: El índice de empleo fue alto. El centralismo, trabajo médico múltiple, modelo centrohospitalario público asistencial y la tendencia a la especialización fueron predominantes.
\end{abstract}

Médicos recién graduados; médicos; empleos en salud; recursos humanos en salud.

\begin{abstract}
Working characteristics of young physicians graduated from San Fernando School of Medicine

Abstract

Objective: To determine the working characteristics of young physicians graduated from San Fernando School of Medicine. Design: Observational, transversal study. Setting: San Fernando School of Medicine, Universidad Nacional Mayor de San Marcos. Participants: Physicians graduated from the School of Medicine. Interventions: Information about sociodemographics, work performance, professional degree, satisfaction level and extracurricular performance during pregrade was collected directly, by phone interview or by e-mail questionnaire from 232 randomly selected physicians graduated between 1994 and 2003.
\end{abstract}

* Basado en la tesis para optar el título profesional de Médico Cirujano "Características Laborales del Egresado de la E.A.P. Medicina Humana de la UNMSM. 1994-2003" Autor: Huamán Joo M. Asesor: Medina Verástegui J. Facultad de Medicina Humana, UNMSM. Lima, Perú.

1 Médico Egresado de la Facultad de Medicina Humana, Universidad Nacional Mayor de San Marcos (UNMSM). Lima, Perú.

2 Departamento de Medicina Preventiva. Facultad de Medicina Humana, UNMSM. Lima, Perú.
We also obtained pregrade final scores. Main outcome measures: Working rate, labor type, work performance, especialization. Results: From all subjects 72,4\% were men, 73,2\% lived in Lima, 19,3\% lived outside of Lima and 7,5\% abroad; $97 \%$ had remunerated work, 51,4\% had two or more jobs; $71 \%$ worked for public institutions. The predominant kind of work was clinical; $78,8 \%$ reached the required work performance; $64,9 \%$ was specialized; $15,4 \%$ had or studied for a master degree. No relationship between pregrade factors and work or professional characteristics was found. Conclusions: Working rate was high. Centralism, multiple jobs, clinical care in public hospitals and tendency to become specialized were predominant.

Key words: Physicians, junior; physicians; health occupations; health manpower.

\section{INTRODUCCIÓN}

El proceso de autoevaluación en las escuelas médicas busca el mejoramiento continuo de la institución, para alcanzar la cali- 
dad y excelencia determinada por estándares nacionales e internacionales $\left({ }^{1,2}\right)$. Este proceso es organizado y conducido por sus propios integrantes, siendo pieza fundamental en el desarrollo sostenido de toda Escuela (13). Al inicio del presente siglo, la Facultad de Medicina de San Fernando (Universidad Nacional Mayor de San Marcos - UNMSM) elaboró y ejecutó un agresivo programa de autoevaluación, con la finalidad de lograr su acreditación $\left({ }^{4}\right)$. No obstante haberse logrado dicho objetivo, este proceso debe mantenerse, teniendo en cuenta los acelerados y progresivos cambios en la enseñanza médica $\left(^{5-8}\right)$ y la amenaza en que se constituyen las otras 27 facultades de medicina existentes a nivel nacional $\left({ }^{9}\right)$.

Dentro de este proceso de autoevaluación, es importante determinar cómo se desenvuelve el médico joven egresado de nuestra Facultad, pues refleja qué tan adecuado es el 'producto final' de San Fernando, en el 'mercado de salud' $\left({ }^{10}\right)$. Más aún, la importancia del recurso humano en los sistemas de salud $\left({ }^{11}\right)$ coloca al médico en el centro del escenario, como miembro líder de los equipos de salud multidisciplinarios y a la Universidad como responsable directa de su formación profesional $\left({ }^{12}\right)$.

Existen varios estudios acerca del ejercicio profesional médico en el país $\left(^{12-21}\right)$. Recientemente, el Instituto de Desarrollo de Recursos Humanos (IDREH) presentó una publicación al respecto, concluyendo que existe sobrepoducción de profesionales, centralismo, tendencia a la especialización y subespecialización, predominancia del modelo hospitalario asistencial, crecimiento progresivo del sector salud privado y del fenómeno de emigración, conflictividad laboral, entre otras características $\left({ }^{12}\right)$; lo que genera un contexto de difícil inserción y alta competitividad para el médico recientemente egresado. No existen datos previos acerca del perfil laboral del egresado de la $\mathrm{Fa}-$ culta de Medicina San Fernando, y es inde- bido considerar que este perfil nacional refleja lo que sucede con los egresados de cada Escuela de Medicina, especialmente en los primeros 10 años de ejercicio profesional.

Por otro lado, algunos investigadores han relacionado factores -como el género y calidad de educación médica- con el desempeño laboral y profesional posterior $\left({ }^{22,23}\right)$. Más aún, durante mucho tiempo se ha considerado que existe una relación directa entre las calificaciones curriculares y el éxito laboral; sin embargo, no existen investigaciones que validen dicha afirmación en nuestro medio.

Motivados por esta situación, nos planteamos realizar esta investigación con el objetivo de determinar las características laborales del médico joven egresado de San Fernando y explorar si existe algún factor curricular o extracurricular del pregrado que se relacione al éxito laboral.

\section{MATERIALES Y MÉTODOS}

Se realizó un estudio observacional, transversal, en una muestra de 232 médicos egresados de la Escuela Académico Profesional (EAP) de Medicina Humana San Fernando, de la UNMSM, entre 1994-2003. La selección de los sujetos fue aleatoria sistemática, a partir de un listado de 2337 egresados proporcionado por la Unidad de Matrícula de la Facultad de Medicina de la UNMSM.

Con el objetivo de ubicar a los sujetos de estudio, se obtuvo los teléfonos, correo electrónico y/o dirección del centro de labores, mediante datos disponibles en la Unidad de Matrícula de la Facultad de Medicina UNMSM, Colegio Médico del Perú, página web de las respectivas promociones, guía telefónica y/o mediante información brindada por sus compañeros de promoción colaboradores del estudio. Una vez ubicados los sujetos de estudio, la información se reco- 
lectó mediante entrevista directa o vía telefónica. En el caso de contacto vía correo electrónico, se envió un cuestionario (versión plantilla de MS-Word 2000). El período de aplicación del cuestionario abarcó desde el mes de setiembre de 2004 hasta agosto de 2005. La entrevista o cuestionario tuvo como objetivo recolectar datos relacionados a las siguientes variables: edad, sexo, estado civil, lugar de nacimiento, lugar de procedencia, educación secundaria, actividad laboral, tipo de actividad laboral, número de trabajos, lugar de trabajo, actividad laboral esperada según la formación del pregrado, grado profesional (especialización y grado académico), nivel de satisfacción profesional, familiar de primer grado médico; variables del pregrado: estudio en instituto superior, estudio en idiomas, ayudantías, externado, estudios en el extranjero, pertenencia a agrupaciones extracurriculares.

Se obtuvo las notas finales de los sujetos de estudio de la base de datos de la Unidad de Matrícula de la Facultad de Medicina San Fernando.

Los criterios de inclusión de los sujetos del estudio fueron: que fuera ubicado mediante alguna de las modalidades anteriormente descritas, que aceptara responder la entrevista o resolver el cuestionario. Los criterios de exclusión fueron: que la entrevista o el cuestionario hubiera sido respondido de forma incompleta, que no se encontrara la nota final del pregrado en la base de datos de la Unidad de Matrícula de la Facultad de Medicina de San Fernando.

El procesamiento y análisis de datos se realizó en Microsoft Excel 2000 y el programa estadístico SPSS v. 12 y comprendió análisis de frecuencias para variables cualitativas, comparación de frecuencias y aplicación de chi $^{2}$ y Anova, análisis de medias para variables cuantitativas, comparación de medias y aplicación de t-student.

\section{RESULTADOS}

Dentro de las características sociodemográficas, de los 232 egresados incluidos en el estudio, $4(1,4 \%)$ habían fallecido. Los varones representaron el $72,4 \% ; 53,5 \%$ de los médicos estaba casado. Entre los egresados 1994 - 1996, 76,7\% se encontraba casado, mientras que, entre los egresados 2001- 2003, 20,3\% estaba casado. El 73,2\% radicaba en Lima, $19,3 \%$ en provincias y $7,5 \%$ en el extranjero (Tabla 1).

Entre los egresados que radicaban en la capital, 43,5\% procedía de Lima Moderna,

Tabla 1. Caracteristicas demográficas de los médicos egresados de San Fernando 1994- 2003.

\begin{tabular}{|c|c|c|c|c|c|c|c|c|c|c|}
\hline \multirow[t]{2}{*}{ Egresado } & \multirow{2}{*}{$\begin{array}{c}\mathrm{N}^{\circ} \\
\text { Total }\end{array}$} & \multirow{2}{*}{$\begin{array}{c}\mathrm{n}^{\circ} \\
\text { Muestra }\end{array}$} & \multirow[t]{2}{*}{ Edad } & \multicolumn{2}{|c|}{ Sexo } & \multicolumn{2}{|c|}{ Estado civil } & \multicolumn{3}{|c|}{ Procedencia } \\
\hline & & & & Masculino & Femenino & Soltero & Casado & Lima & Provincia & Extranjero \\
\hline 1994 & 210 & 22 & 36,8 & 15 & 7 & 5 & 17 & 17 & 3 & 2 \\
\hline 1995 & 264 & 27 & 36,5 & 21 & 6 & 6 & 20 & 23 & 3 & 1 \\
\hline 1996 & 266 & 26 & 34,5 & 22 & 4 & 6 & 19 & 15 & 6 & 3 \\
\hline 1997 & 306 & 29 & 33,9 & 20 & 9 & 7 & 20 & 20 & 5 & 2 \\
\hline 1998 & 154 & 16 & 33,1 & 11 & 5 & 5 & 11 & 12 & 2 & 2 \\
\hline 1999 & 154 & 15 & 32,1 & 8 & 7 & 8 & 7 & 13 & 2 & 0 \\
\hline 2000 & 317 & 28 & 32,0 & 20 & 8 & 14 & 14 & 18 & 7 & 3 \\
\hline 2001 & 232 & 25 & 30,1 & 22 & 3 & 17 & 8 & 14 & 7 & 4 \\
\hline 2002 & 187 & 19 & 29,1 & 13 & 6 & 15 & 4 & 13 & 6 & 0 \\
\hline 2003 & 247 & 25 & 27,8 & 17 & 8 & 23 & 2 & 22 & 3 & 0 \\
\hline Total & 2337 & 232 & 32,6 & 169(72,4\%) & $63(27,6 \%)$ & $106(53,5 \%)$ & 122(46,5\%) & $167(73,2 \%)$ & $44(19,3 \%)$ & $17(7,5 \%)$ \\
\hline
\end{tabular}


$18,3 \%$ de Lima Norte, 15,6\% de Lima Este, $14,5 \%$ de Lima Antigua, 4,3\% del Callao y $3,8 \%$ de Lima Sur.

Dentro de las características laborales, 97\% poseía al menos un trabajo remunerado. El restante $3 \%$, que no trabajaba, pertenecía a las promociones egresadas 2002 y 2003. De los 221 egresados que laboraba, $48,6 \%$ poseía un trabajo remunerado, $29,4 \%$ tenía 2 trabajos y $22 \%$ indicaba tener 3 o más trabajos. El promedio de trabajos remunerados se encontraba directamente relacionado con el año de egreso (Figura 1).

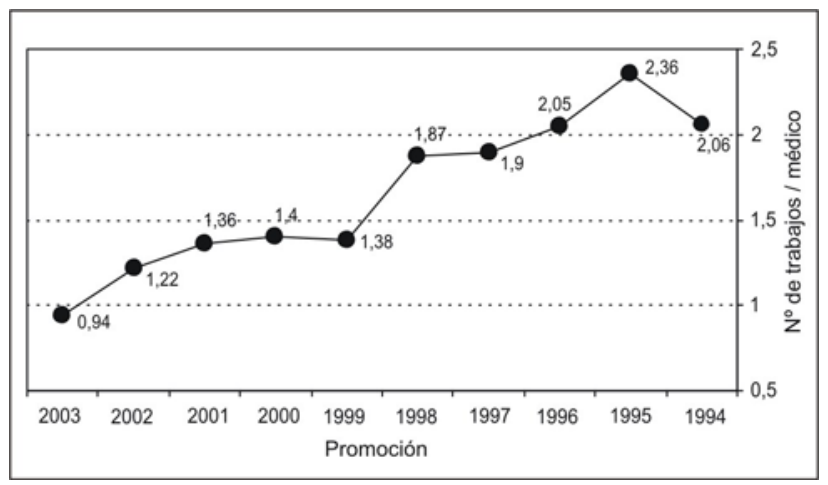

Figura 1. Número de trabajos por cada médico, según año de egreso.
El $72,4 \%$ de los egresados que laboraba lo hacía en Lima, $19,9 \%$ en provincias y $7,7 \%$ en el extranjero. El tipo de labor predominante era asistencial (Tabla 2); sin embargo, $15,5 \%$ de ellos también realizaba docencia y $9,4 \%$, se dedicaba también a salud pública y/o gerencia.

En cuanto al trabajo remunerado al que más horas dedicaba, $29 \%$ de los egresados que trabajaba en el Perú indicó hacerlo en una institución privada, mientras que $43,6 \%$ en entidades del Minsa, $23 \%$ en EsSalud y $4,4 \%$ en las Fuerzas Armadas (Figura 2).

Aquellos que laboraban únicamente en el subsector privado, lo hacían en establecimientos no propios, como personal contratado. El 45,1\% de los egresados que se desempeñaba en el sector público, tenía un segundo trabajo en el sector privado. Dentro de este grupo, que se desempeñaba en el sector público y privado a la vez, $27,8 \%$ lo hacía en un negocio propio, tipo consultorio.

Entre los egresados que trabajaban en el sector público nacional, $40,3 \%$ lo hacía en establecimientos de atención nivel uno y dos, mientras $59,7 \%$ laboraba en nivel de

Tabla 2. Lugar de trabajo y labor predominante de los médicos egresados de San Fernando 1994-2003.

\begin{tabular}{|c|c|c|c|c|c|c|c|c|c|}
\hline \multirow[t]{2}{*}{ Egresado } & \multirow[t]{2}{*}{$\mathrm{n}^{\circ}$} & \multicolumn{3}{|c|}{ Lugar de trabajo } & \multicolumn{5}{|c|}{ Labor predominante } \\
\hline & & Lima & Provincia & Extranjero & Asistencia & Docencia & $\mathrm{Ge} / \mathrm{SP}$ & Investigación & NRS \\
\hline 1994 & 22 & 17 & 3 & 2 & 19 & - & 2 & 1 & - \\
\hline 1995 & 26 & 22 & 3 & 1 & 25 & 1 & - & - & - \\
\hline 1996 & 25 & 16 & 6 & 3 & 24 & - & 1 & - & - \\
\hline 1997 & 27 & 20 & 5 & 2 & 21 & - & 4 & 2 & - \\
\hline 1998 & 16 & 12 & 2 & 2 & 15 & - & 1 & - & - \\
\hline 1999 & 15 & 13 & 2 & - & 14 & - & 1 & - & - \\
\hline 2000 & 28 & 18 & 7 & 3 & 26 & - & 2 & - & - \\
\hline 2001 & 25 & 14 & 7 & 4 & 20 & - & 4 & 1 & - \\
\hline 2002 & 18 & 12 & 6 & - & 14 & 1 & 2 & 1 & - \\
\hline 2003 & 19 & 16 & 3 & - & 8 & 1 & - & - & - \\
\hline Total & 221 & $160(72,4 \%)$ & $44(19,9 \%)$ & $17(7,7 \%)$ & $196(88,7 \%)$ & $3(1,4 \%)$ & $17(7,7 \%)$ & $5(2,2 \%)$ & 0 \\
\hline
\end{tabular}

Ge/SP=Gerencial/Salud Pública, $N R S=$ No relacionado a la Salud . 


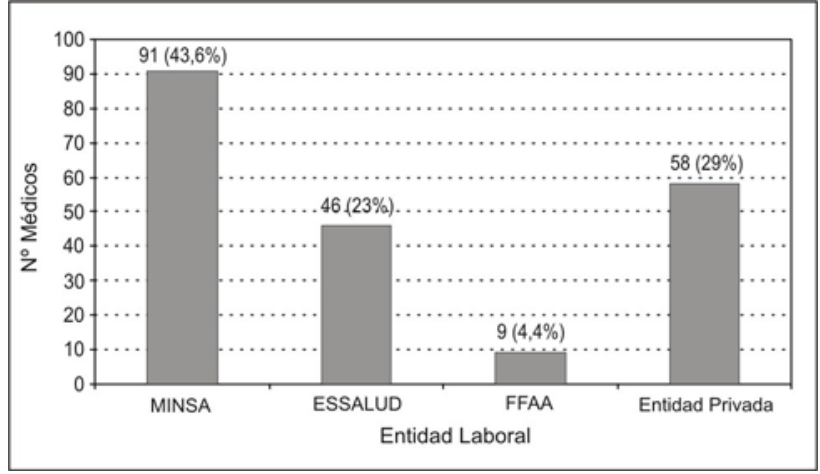

Figura 2. Entidad laboral de médicos egresados San Fernando, 1994-2003.

atención tres. Dentro de este último grupo, $55,8 \%$ se encontraba trabajando en estos hospitales como residente. Si dejamos de lado al grupo que se encontraba haciendo la residencia, el $60,4 \%$ se encontraría en niveles de atención uno y dos, mientras $39,6 \%$ estaría en entidades nivel tres.

El 78,8\% afirmó haber alcanzado el desempeño laboral esperado, según la formación del pregrado (Tabla 3).

Sobre la especialización y el grado académico, 39,5\% de los egresados era especialista, mientras $35,1 \%$ no se había especializado. El 25,4\% restante se encontraba en la residencia médica, en proceso de especialización. En cuanto al grado académico, $15,4 \%$ de los egresados poseía o estaba culminando una maestría, mientras $2,6 \%$ había realizado o estaba culminando el doctorado.

El $79 \%$ se encontró satisfecho de su grado de especialización, mientras que $64,1 \%$ refirió estar satisfecho del grado académico alcanzado.

De las características del pregrado, el promedio final del pregrado entre los participantes del estudio fue 14,62 $( \pm 0,92)$. En cuanto a las actividades extracurriculares dentro del pregrado, $78 \%$ de los egresados estudió al menos un idioma (79,4\% inglés, $12,2 \%$ protugués, $6,9 \%$ francés). El 8,8\% de los egresados estudió alguna carrera superior técnica (75\% relacionada a la salud). El 29,4\% de los egresados participó en alguna ayudantía durante el pregrado, 66,2\% participó en externado voluntario. A su vez, $52,2 \%$ participó de alguna agrupación tipo Sociedad Científica de San Fernando, grupos socioculturales, de proyección a la comunidad o asociación deportiva.

Dentro de los factores relacionados al desempeño laboral, los casados tenían mayor número de trabajos $(1,90 \pm 0,777)$ que los solteros $(1,48 \pm 0748)(p=0,000)$. A su vez, existió relación entre el lugar de nacimiento y el lugar de trabajo $(p=0,014)$, siendo más frecuente haber nacido en provincia entre aquellos que laboraban en provincia. No existió diferencias significativas según el sexo en alguna de las características laborales estudiadas.

Aquellos que trabajaban en el extranjero, habían logrado el desempeño laboral esperado en mayor proporción que aquellos que trabajaban en Lima y provincia $(p=0,000)$. Del mismo modo, los egresados que trabajaban en el área asistencial habían alcanzado en mayor proporción el desempeño esperado, comparados con los médicos que laboraban en otras áreas $(p=0,003)$.

Entre los egresados que trabajaban en Lima, aquellos que se desempeñaban en el sector público afirmaban haber alcanzado en mayor proporción el desempeño esperado, en comparación con aquellos que laboraban en el sector privado $(p=0,028)$. Den-

Tabla 3. Percepción del egresado acerca de haber alcanzado el desempeño laboral esperado.

\begin{tabular}{lccccc}
\hline \multirow{2}{*}{ Egresados } & Insatisfecho & \multicolumn{4}{c}{ Satisfacción } \\
\cline { 3 - 6 } & & Poco & Regular & Buena & Máxima \\
\hline $1994-1996$ & $3,4 \%$ & $1,7 \%$ & $6,9 \%$ & $20,7 \%$ & $67,3 \%$ \\
$1997-1999$ & - & - & $19 \%$ & $30,9 \%$ & $50,1 \%$ \\
$2000-2003$ & $10,7 \%$ & - & $17,2 \%$ & $14 \%$ & $58,1 \%$ \\
Total & $6,2 \%$ & $0,5 \%$ & $14,5 \%$ & $19,7 \%$ & $59,1 \%$ \\
\hline
\end{tabular}


tro del sector público, los que más destacaban en llegar al desempeño esperado eran aquellos que laboraban en EsSalud, en comparación a sus colegas del Minsa y Fuerzas Armadas $(p=0,015)$.

Tener algún familiar de primer grado médico se relacionó de forma significativa con laborar en el extranjero $(p=0,024)$ y laborar en el sector privado $(p=0,006)$.

No se encontró relación significativa entre la nota final del pregrado y lograr el desempeño laboral deseado $(p=0,436)$. Entre los médicos egresados en el 2003, no se encontró diferencia significativa $(p=0,211)$ entre los promedios finales del pregrado de los que tenían trabajo vs. aquellos que no tenían trabajo. Tampoco existió relación entre las características del pregrado estudiadas y el desempeño laboral posterior.

En cuanto a los factores relacionados a la especialización y grado académico, no se encontró relación estadísticamente significativa entre los promedios finales del pregrado y el grado de especialización $(p=0,918)$ o el grado académico $(p=0,102)$. Excepto el haber participado en el externado voluntario $(p=0,011)$, no existió relación significativa entre las características del pregrado estudiadas y el grado de especialización.

Se encontró que aquellos que cursaban o habían terminado alguna especialidad se encontraban más satisfechos que aquellos no especialistas $(p=0,000)$. Sin embargo, el tener un mayor grado académico no se relacionaba con mejor nivel de satisfacción $(p=0,6)$.

\section{DISCUSIÓN}

Nuestro estudio demuestra que $97 \%$ de los médicos jóvenes egresados de la Facultad de Medicina San Fernando se encontra- ba trabajando, lo que dista del 7,6\% de desempleo médico a nivel nacional. Esto estaría en relación a una mayor proporción de médicos desempleados provenientes de otras universidades. La UNMSM continúa siendo la mayor proveedora de médicos en el país $(43,2 \%)$, seguida por la Universidad Federico Villarreal, con $10,6 \%\left({ }^{21}\right)$. Excepto departamentos, como Ica, La Libertad, Arequipa, Moquegua, Puno, Tacna; la UNMSM es también aportadora de la mayoría de profesionales médicos en provincias, lo que demuestra la importancia del médico sanfernandido a nivel nacional. Es destacable que, 4 años después del egreso, todos se encontraban trabajando en actividades relacionadas a la salud, lo que indicaba una adecuada inserción en el mercado de salud.

A pesar de que la totalidad de egresados encuestados que trabajaba refirió hacerlo en labores relacionadas a la salud, no se pudo determinar la magnitud real del fenómeno de subempleo en la población estudiada. Se necesita establecer con exactitud las competencias específicas del médico general y especialista para según éstas evaluar cada subconjunto de ocupaciones relacionadas a la salud, teniendo en cuenta aspectos remunerativos, sobretodo aquellas incluidas en el subsector privado, que es el más expuesto al riesgo de precarización $\left({ }^{12}\right)$. Una aproximación a la cantidad de subempleo podría reflejarse en el $6,7 \%$ y $14,5 \%$ de egresados del estudio que respectivamente refirieron no haber alcanzado o haber alcanzado parcialmente el desempeño laboral esperado, según la formación del pregrado.

El perfil académico profesional del médico sanfernandino no es explícito en indicar que la Universidad forma a un médico para el Perú. Sin embargo, las características de los objetivos curriculares y plan de estudios así lo indican $\left({ }^{24}\right)$. Esta es una meta en gran parte satisfecha si tomamos en cuen- 
ta que $19,3 \%$ se encontraba en provincia. La UNMSM, al ser una entidad estatal, no considera dentro de sus objetivos formar médicos con perspectiva de emigrar al extranjero. Sin embargo, el $7,5 \%$ de los egresados ha emigrado a distintos países de América y Europa. Esto es entendible, debido al escenario nacional de salud de alto grado de competitividad, sobresaturado de profesionales médicos $\left({ }^{12,21}\right)$, la tendencia actual de médicos de países latinoamericanos a la migración $\left({ }^{25-27}\right)$ y el fenómeno de globalización $\left({ }^{8}\right)$. En este sentido, es importante la estrategia de nuestra Facultad en reducir sistemáticamente la cantidad de plazas anuales de ingreso e impulsar los programas de cooperación internacional. Estos programas deben ser implementados en los próximos años, de tal manera que faciliten el perfeccionamiento del profesional médico, pero a su vez eviten la 'fuga de talentos' al extranjero, mediante un compromiso de retorno o retribución.

No estudiamos directamente el grado de satisfacción económica del egresado sanfernandino. Sin embargo, observamos que $51,4 \%$ indicó tener 2 o más trabajos y que de éstos la mayoría se desempeñaba en el sector público y privado a la vez. Todos los estudios previos indican la existencia del trabajo médico múltiple, esto es, la simultaneidad de varios trabajos $\left({ }^{12}\right)$, lo que reflejaría la falta de satisfacción económica del médico en el Perú. Esto va en relación a lo presentado por Lip, quien indica que $55,5 \%$ de los médicos tenía remuneraciones mensuales menores a los 2000 soles $\left({ }^{21}\right)$. Por otro lado, es interesante observar que $43,5 \%$ de los sujetos del estudio que radicaban en la capital provenía de Lima Moderna, zona que alberga a $98,6 \%$ de la población del sector socioeconómico A y a $52,9 \%$ del sector socioeconómico B $\left({ }^{28}\right)$. Los distritos con mayores índices de pobreza y pobreza extrema se encuentran en Lima Sur y Lima Norte, donde radicaban el $18,3 \%$ y $3,8 \%$ de egresados, respectivamente.
En cuanto al trabajo remunerado al que más horas se le dedicaba, el Minsa fue el principal proveedor de plazas rentadas entre los médicos de San Fernando, con $43,6 \%$, seguido por EsSalud con $23 \%$ y las Fuerzas Armadas con $4 \%$, lo que se aproxima a datos del 2004 a nivel nacional, con $43 \%$ de médicos en el Minsa y $26,1 \%$ en EsSalud. La distribución centrohospitalaria en el sector público sigue siendo importante, incluso entre los médicos jóvenes, esto asociado a que la residencia médica se realiza en centros nivel III.

Se observa en este estudio que $29 \%$ laboraba en el sector privado, lo que demuestra la tendencia del profesional médico a introducirse en la práctica privada $\left({ }^{19,21}\right)$. Destaquemos además que $45,1 \%$ de los egresados que se desempeñaba en el sector público tenía un segundo trabajo en el sector privado. Conforme aumentan los años de egreso, son más los médicos del sector público que ingresan a la práctica privada, en parte debido a que la residencia demandó dedicación exlusiva al hospital público durante los primeros años.

El subgrupo de médicos sanfernandinos que trabajaba por cuenta propia o autónoma dentro del sector privado del sistema de salud $(27,8 \%)$ fue menor al publicado a nivel nacional, en 1996 , en que $38,1 \%$ de los médicos que trabajaban para el Minsa indicó laborar en 'consultorio privado propio'(20). Más aún, no se encontró en la población de médicos que se dedicaran al negocio propio como único trabajo remunerado, lo que se relaciona a la falta de facilidades que el Estado y los organismos rectores de salud brindan a la pequeña y microempresa; además del monopolio que las grandes clínicas y Entidades Prestadoras de Salud (EPS) vienen estableciendo en este sector.

Encontramos que $78,8 \%$ de los egresados refirió haber alcanzado el desempeño laboral esperado, según la formación del 
pregrado, lo que grafica tanto el aceptable grado de satisfacción del egresado sanfernandino con respecto a su actividad laboral como la adecuada formación recibida en el pregrado para alcanzar este objetivo. Esto contrasta con los niveles de satisfacción del alumno sanfernandino en sus primeros años de estudio, en que señalaba las limitaciones de los cursos de ciencias básicas impartidos en la Facultad $\left({ }^{29}\right)$.

En comparación con el Minsa, aquellos que laboraban en EsSalud habían alcanzado en mayor proporción el desempeño laboral esperado, según la formación del pregrado. Esto podría estar en relación a mayor disponibilidad de recursos y beneficios en EsSalud, así como a diferencias salariales. El ingreso remunerativo promedio, en el 2005, para un médico de EsSalud fue de S/. 3 375, mientras que en el Minsa fue S/. $2328\left({ }^{12}\right)$.

Solo $2,2 \%$ de egresados se dedicaba principalmente a la investigación, lo que refleja una debilidad de nuestra Escuela. El Perfil Académico Profesional presenta al médico como un investigador $\left({ }^{23}\right)$; más aún, las perspectivas de cambio y mejoramiento en educación médica a nivel mundial señalan a la investigación como pilar indispensable de toda Escuela de Medicina del siglo XXI $\left({ }^{2,5-8}\right)$. Esto hace necesario un mayor incentivo de la investigación en el pregrado, pues aún representa una debilidad de nuestra Escuela, reflejada en estudios previos $\left({ }^{30,31}\right)$. Por otro lado, no se conoce cuál es la real oferta de trabajo en investigación a nivel nacional, por lo que este campo amerita un estudio situacional detallado.

Los egresados que se dedicaban principalmente a la docencia representaban el $1,4 \%$; sin embargo, $15,5 \%$ de los egresados que se dedicaba a la labor asistencial también realizaba docencia. Esto demuestra la creciente oferta de trabajo en educación médica, consecuencia del aumento de fa- cultades e institutos educativos relacionados a la salud, a nivel nacional.

La tendencia a la especialización y subespecialización es un fenómeno ampliamente estudiado $\left({ }^{12,19,21,32}\right)$. Mientras que la proporción de médicos que se declaraba especialista fue $32 \%$ en 1957 , en 1964 fue $73 \%$, en $1988,72 \%$, y en 1996, 73,4\%. Esto se correlaciona con el $39,5 \%$ de egresados especialistas y $25,4 \%$ en curso de especialización que presenta este estudio. San Fernando forma a cerca del $60 \%$ de especialistas a nivel nacional y casi $35 \%$ de los postulantes al residentado médico de la UNMSM, en el 2003, pertenecía a su Escuela, lo que refleja el interés del egresado por continuar su formación de posgrado en el Alma Mater $\left({ }^{33}\right)$. A pesar de lo mencionado, este estudio indica que el ser especialista no se relaciona con alcanzar el desempeño laboral deseado, pero sí se relaciona a mayor nivel de satisfacción de objetivos profesionales y acceso a nivel de atención III. Radicar en provincia limita la especialización, lo que podría influir en la decisión de ejercer la profesión fuera de la capital.

El obtener mejores promedios curriculares finales, haber estudiado en centro de idiomas, tener estudios en instituto superior, haber participado en ayudantías o en agrupaciones extracurriculares de diversa naturaleza no influyen en el desempeño laboral ni profesional posterior. Esto cuestiona los modelos de autoevaluación planteados por diversos estudios, en que factores del pregrado como estos sirven de indicadores acertados al calificar una Facultad de Medicina $\left({ }^{3}\right)$, pues no representan ventajas competitivas a posteriori. Debería revisarse el método de evaluación curricular vigente, de modo que permita disminuir la brecha existente entre la realidad del pregrado y el desenvolvimiento laboral y profesional del egresado.

Algunos factores sociodemográficos demostraron tener influencia en el desempe- 
ño laboral y profesional. Tener familiares médicos se relacionó a mayores oportunidades de trabajo en el sector privado y el extranjero. Es interesante observar que $43,9 \%$ de los egresados tenía algún familiar cercano que era médico, lo que indicaba la persistencia de la tradición médica en algunas familias. Por otro lado, no encontramos diferencia significativa según sexo en las características laborales, profesionales y académicas estudiadas; a pesar de lo planteado por Durán-Arenas en México, quien señala diferencias de género en cuanto al estatus profesional médico $\left({ }^{22}\right)$. Esto hace que el incremento progresivo de mujeres estudiando medicina no represente cambios en el perfil laboral o profesional.

Encontramos algunas limitaciones al ejecutar esta investigación. Hubo 52 egresados que resultaron excluidos del estudio, 37 al no ser ubicados y 15 al negar su participar. Esto representa un $19,4 \%$ de exclusión, lo que podría generar un factor de error en los resultados. El tipo de estudio hace difícil obtener porcentajes de exclusión más bajos en muestras tomadas al azar. Por ejemplo, Saco y Villavicencio recolectaron 109 encuestas de las 147 previstas en su estudio en la UNSAAC, lo que representó al $74,15 \%$ de su universo $\left({ }^{34}\right)$. Se necesita elaborar mejores estrategias para la inclusión de sujetos en este tipo de trabajos. En ese sentido, la propia Escuela, a través de su capacidad instalada y los vínculos formales que mantiene con los egresados, debería conducir en el futuro los análisis situacionales laborales y profesionales, como parte de su proceso de autoevaluación y mejoramiento continuo.

En conclusión, existió un alto índice de empleo, centralismo, trabajo médico múltiple, modelo centrohospitalario público asistencial y tendencia a la especialización, como las caracerísticas laborales predominantes entre los médicos jovenes egresados de la Facultad de Medicina de San Fernando.

\section{REFERENCIAS BIBLIOGRÁFICAS}

1. Consorcio de Universidades. Autoevaluación Institucional. Manual para instituciones de educación superior. Lima: Pontificia Universidad Católica del Perú; 1999.

2. Lanosa GA. La excelencia en la enseñanza de la medicina. Pren Méd Argent. 1997;84:328-31.

3. Cardozo O, Lizarraga A, Raimondo R. Evaluación longitudinal en la carrera de medicina de la Universidad Del Salvador. Pren Méd Argent. 1997;84:652-6.

4. Piscoya J. Acreditación de facultades de medicina y su impacto en la formación de recursos humanos en salud. An Fac Med Lima. 1998;59(3):232-43.

5. Binia A. Tendencias modernas en educación médica. Bol Acad Nac Med B Aires. 1994;72:333-48.

6. Cuellar Z. Panorama de las academias frente al siglo XXI. Bol Acad Nac Med B Aires. 1997;187-201.

7. La educación médica peruana al inicio del tercer milenio. An Fac Med Lima. 1998;59(3):213-4.

8. Casas J. Educación médica en el Perú en la perspectiva del nuevo milenio. Diagnóstico. 2000;39(3):158-65.

9. Berríos J. Resumen y recomendaciones, vertidas en el marco de la $21^{\circ}$ sesión ordinaria de la Academia Nacional de Medicina sobre la acreditación. Comisión para la Acreditación de Facultades o Escuelas de Medicina. Lima, Perú. 2005 Disponible en http://www.cafme.gob.pe/acredita.php? action $=$ fullnews\&id $=10$

10. Cardozo O, Lizarraga A, Raimondo R. Evaluación longitudinal en la carrera de medicina de la Universidad del Salvador. Prensa Médica Argentina. 1997;84:652-6.

11. MINSA-IDREH. Metodología para la formulación de perfiles de competencias para trabajadores del primer nivel de atención. Lima: MINSA-IDREH; 2005.

12. MINSA-IDREH. Situación y Desafíos de los Recursos Humanos en Salud. Lima: MINSA-IDREH; 2005.

13. Universidad Nacional Mayor de San Marcos, Facultad de Medicina Humana. Encuesta médica y hospitalaria en el Perú (2do informe). Lima: Facultad de Medicina UNMSM; 1957.

14. Hall T. Recursos Humanos de Salud. Ministerio de Salud Pública y Asistencia Social. Lima: Oficina Sectorial de Planificación de Salud; 1974.

15. Alarcón J, Reyes N, Piscoya J. Situación de trabajo y características socioeconómicas del médico en Lima Metropolitana. Lima: OPS-Colegio Médico del Perú; 1989.

16. Brito P, Lazo O, Lip C. El Trabajo Médico en el Perú. Lima: OPS-Universidad Particular Cayetano Heredia; 1989.

17. Ministerio de Salud, Oficina de Estadística e Informática. Segundo Censo de Infraestructura Sanitaria y Recursos del Sector Salud. Lima: MINSA; 1996.

18. Swayne J. Fuerza de Trabajo [Documento de Trabajo]. Dirección General de Desarrollo de Recursos Humanos ENSAP. Lima: MINSA; 1996.

19. Escalante G. La formación profesional y el trabajo en salud: el verdadero desarrollo del potencial humano. An Fac Med Lima.1998;59(3):249-55. 
20. Bustíos C, Swayne J. Situación de la Fuerza de Trabajo Médico en el Perú. Escuela Nacional de Salud Pública. Lima: MINSA; 1998.

21. Lip C, Vargas A, Zevallos W, Longa J, Hurtado J. Situación del profesional médico cirujano a ocho años de iniciada la reforma de la salud y la seguridad social. An Fac Med Lima. 2000;61(3):99-124.

22. Durán L. Determinantes del estatus profesional de los médicos en México. Gac Méd Méx. 2001;137(6):509-20.

23. Sewell W, Hauser R. Education, occupation and earnings: achievement in the early career. New York: Academic Press; 1975.

24. Universidad Nacional Mayor de San Marcos, Facultad de Medicina Humana, Escuela Acdémico Profesional de Medicina Humana. Perfil Académico Profesional. Plan Curricular y Objetivos curriculares. Lima: Facultad de medicina UNMSM; 2004.

25. Mejía A. Migration of physicians and nurses: a world-wide picture. Intern J Epidemiol. 1978;7:207-15.

26. Bach S. Migration pattern of physicians and nurses: still the same story? Bull World Health Organization. 2004;82(8):6245.

27. Stilwell B, Diallo K, Zurn P, Vujicic M, Adams O, Dal Poz M. Migration of health-care workers from developing countries: strategic approaches to its management. Bull World Health Organization. 2004;82(8):595-600.

28. Apoyo Opinión y Mercado S.A. Perfiles Zonales de Lima Metropolitana 2005. Lima: Apoyo Opinión y Mercado S.A.; 2005.

29. Guere I, Huamán M, Huauya C, Hurtado F, Heredia I, Huancco J, et al. Análisis de los Planes Curriculares de los tres primeros años de estudio de la Escuelas de Medicina de la UNMSM y la UPCH, 2000. Lima: UNMSM; 2001.

30. Sogi C, Perales A, Anderson A, Bravo E. El proceso de producción científica de los investigadores de la Facultad de Medicina, UNMSM. An Fac Med Lima. 2002;63(2):11524.

31. Sogi C, Perales A. Anderson A, Savala S. Calidad de la producción científica de los investigadores de la Facultad de Medicina, UNMSM. An Fac Med Lima. 2003;64(2):1128.

32. Valdivieso V. La certificación de especialistas en la medicina chilena. Bol Acad Nac Med B Aires. 2001;79:443-52.

33. Manuel C. Proceso de admisión 2003 a Residentado Médico UNMSM. Estudio descriptivo del perfil del postulante. An Fac Med Lima. 2003;64(3):52-8.

34. Saco S, Villavicencio E. Situación actual y perspectivas de los médicos egresados de las ocho primeras promociones de la Facultad de Medicina de la Universidad Nacional de San Antonio de Abad del Cuzco (1989 - 1994). Situa. 1999;14:14-23.

Manuscrito recibido el 05 de marzo de 2007 y aceptado para publicación el 28 de marzo de 2007.

\section{Correspondencia:}

Moisés Huamán Joo

Facultad de Medicina, UNMSM.

Av. Grau 750. Lima 1, Perú.

Correo-e:mhuamanjoo@hotmail.com 